\title{
AUTOMATIC REGULATION OF HEATING IN ELECTRICAL HOT AIR STERILIZATION OF SURGICAL EYE INSTRUMENTS AND SILKS FOR SUTURE*
}

\author{
BY \\ S. Holth \\ OSLO, NORWAY
}

THE ordinary boiling in water or in a two per cent. alkaline solution of sodium carbonate must be done immediately before each operation, but, nevertheless, spoils the edge of instruments made of the good old steel, and also of those made from the best non-corrosive steel. In the later catalogues from factories making surgical instruments, one will find all kinds of pincers, and likewise coarser scalpels and scissors made of non-corrosive steel, and also obstetrical instruments. Hypodermic needles are made of this material; they can tolerate a short heating in the flame. Cutting instruments for operations on the eye in rust-proof steel, which were announced ten years ago, are not seen in the catalogues -a single one, however, excepted.

Professor Johan Hjort (Oslo), in 1898, instructed his assistant to hold the cataract knife in boiling water one minute to spare the edge. The same procedure is adhered to until this day in the large Eye Clinics in London, only with the difference that the knife first is rinsed for one minute in absolute alcohol or in acidum carbolicum liquefactum.

In a lesson during the spring of $1899, \mathrm{E}$. Roux in the Pasteur Institute in Paris said that boiling once for 30 minutes kills all sporeless bacteria, but not the spores of the spore-bearing bacteria. The latter were first killed after an uninterrupted boiling of from two to five hours duration or through interrupted boiling for three days, 15 minutes every day with complete cooling between boiling. Young spores cultures-air-dried at ordinary room temperature-were, however, killed in a single heating in dry air at a temperature of $150^{\circ} \mathrm{C}$. I, myself, could control this fact with spores from anthrax, tetanus and bacillus subtilis. At the same time I could show that excellent cataract knives and keratomes, from A. Luër in Paris, after heating had an edge and a point as sharp as if they had just arrived from the instrument maker.

The firm, E. Adnet et Cie, had made the gas heated doublemantled air ovens of copper. In January, 1900, I obtained a specimen of this oven for the University Eye Clinic and another

- Read in the Norwegian Medical Society, December 21, 1932. 
for the Catholic Hospital of Oslo. In a perforated stopper in the top of the oven a thermometer was introduced, measuring from $50^{\circ}$ to $200^{\circ} \mathrm{C}$. As soon as the temperature had reached $150^{\circ} \mathrm{C}$. the gas burner had to be slightly closed, if the temperature showed any tendency to rise, and opened if it should start falling. This half-hour's watch over the thermometer was carefully carried out by the attendants of both hospitals. It had to be continued also after the introduction several years ago of the electrically heated ovens of Lautenschläger with periodical breaking and closing of the current under close observation of the thermometer.

As far back as January, 1901, the instrument maker, Moritz Gallus, lamented the strongly declining sharpenings of used cataract knives from the University Eye Clinic and the small sale of new ones, a fact I held to be a practical compliment to the introduction of the dry air sterilization, which successively has been adopted almost exclusively by all operators in the Catholic Hospital in Oslo. For this sterilization the instruments are placed closely together in dry metal cases and should be packed in two layers of filtering paper. During the operation the instruments are placed more conveniently on two dry towels, sterilized in the autoclave. Instruments should not be put into any kind of fluid during the operation. If an eye instrument must be used a second time during the same operation, it should first be rinsed in absolutely sterile water; it is better to have four dry sterilized iris spatulae in the case. One should, for the dry air sterilization, have at least two larger metal cases for typical operations, and at least one smaller metal case for each of the less frequently used special instruments. When the double layer of filtering paper is removed from the case, the sterile instruments are ready for use at any time.

All known pathogenic bacterial spores are killed at $150^{\circ} \mathrm{C}$. within 30 minutes. But there are microbes from dust and soil with spores that are killed only in temperatures of $160^{\circ} \mathrm{C}$. After having convinced myself that the finest cutting instruments of the old instrument steel were unstained after 30 minutes heating of dry air at $160^{\circ}-165^{\circ} \mathrm{C}$., and that their edge and point were as sharp as immediately after sharpening, I asked Dr. med. Th. Thjötta to try this temperature upon the most hardy bacteria spores obtainable.

He chose bacillus mesentericus (Flügge). Thjötta now infected a number of sewing needles and silks with cultures of this microbe, adding to the culture some starch for better adhesion to the smooth surface of the needles. All material was dried in the open air before being enclosed in sterile test tubes plugged with common cotton plugs. Next all tubes were put in the dry air sterilizer and heated for 30 minutes at $160^{\circ} \mathrm{C}$. This treatment killed all 
spores in the tests, as shown by the fact that the plain broth pipetted into the tubes with needles and silks remained sterile for six days in the incubator, while the controls (not heated) gave a good growth.

Beforehand, I found it rather plausible that the silks might be somewhat weakened by $160^{\circ} \mathrm{C}$. and had accordingly first stated that each silken thread had the same strength all through its length. This was done by the use of spring balances (trout balance for the slender conjunctival silk, salmon balance for the heavier skin silks) thus measuring the bursting point of the thread. I was very glad to find that the silks were not weakened by the temperature of $160^{\circ}-165^{\circ} \mathrm{C}$. It even happened that some threads after treatment were harder and stronger, as shown by the fact that the weight sometimes had to be increased about 20 per cent. of the original weight, before the bursting point was reached. Chinese female hairs occasionally used for sutures in the conjunctiva could not be tested after this method as the strength of the hair always differs in different parts of its length. Suitable lengths of silken thread with one or two suture needles fixed to them are enclosed in double filtering paper, are thus sterilized without the points of the needles being blunted - they are ready for use at any time.

The sterilization was carried out by Thjötta in the bacteriological laboratory of the Norwegian Army by means of the dry air sterilizer of W. C. Heraeus (Hanau a. Main) supplied with the auto-regulator for temperatures between $+50^{\circ} \mathrm{C}$. and $+220^{\circ} \mathrm{C}$., patented for this firm three years ago. The firm had taken out a patent January 30, 1913, for a thermoregulator for $70^{\circ} \mathrm{C}$. (three days "Tyndallising procedure"). The constructions regarding higher temperatures began four years ago.

To spare the nurses from the tiring work of watching the mercury thermometer during the sterilization, I asked "Vor Frue Hospital" in June, 1932, to procure the thermoregulator mentioned above and to combine it with the electrically heated hot air sterilizer of Lautenschläger (double walled copper built). This was immediately done. It was mounted in August, and works well.

The whole arrangement from W. C. Heraeus is seen in Fig. 1. A double watled oven is made of aluminium with sheets of asbestos between the two layers of metal. The oven has three boards, where the instrument cases (each wrapped in double filtering paper) can be placed.

Uppermost in the oven is the contact thermometer; this is a bi-metal tube. The lever connection between this one and the thermoscale to the right, cannot be seen on the picture. The hand of the scale can be placed on any of the radial lines between $+50^{\circ}$ and $+220^{\circ} \mathrm{C}$. to the extreme right. Above the oven the very 


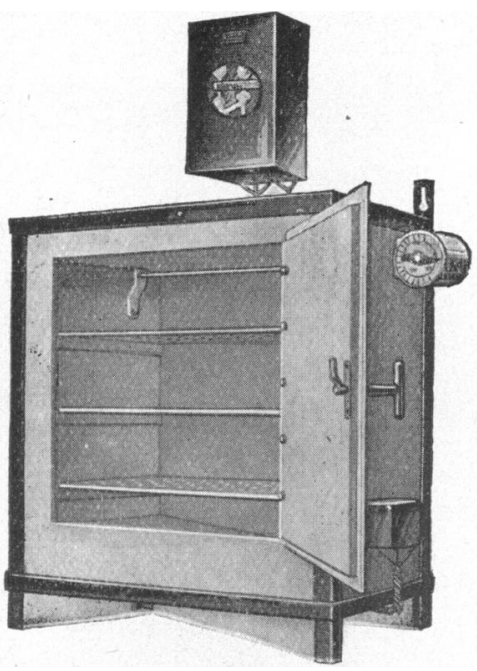

FIG. 1.

Electrically heated dry air sterilizer with contact thermometer and mercurial relay from W. C. Heraeus.

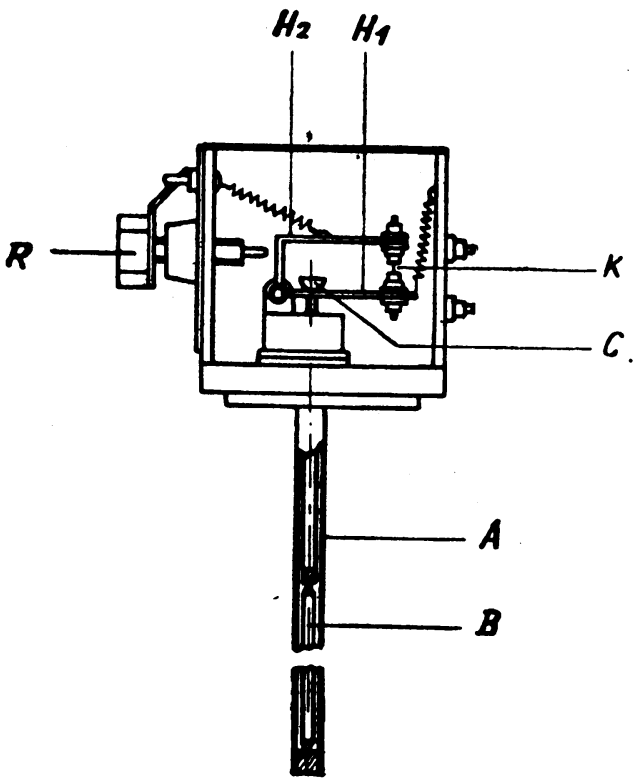

FIG. 2.

Diagram to explain the contact thermometer of W. C. Heraeus. 
important mercurial relay is placed (details in Fig. 3). Further, a common mercurial thermometer with a scale ranging from $+50^{\circ}$ to $+200^{\circ} \mathrm{C}$. is placed through a perforated stopper in the roof of the oven (not seen in the picture). In the hot air sterilizer of Lautenschläger the thermometer is placed before the copper door and the metal protected mercury-container behind the door within the oven.

In Fig. 2 is seen a diagram showing the principle of the contact thermometer, which is made out of two metals.

It consists of an outer metal tube "A," with a large expansion under increasing temperature and an inner "invar" rod " $B$ " with

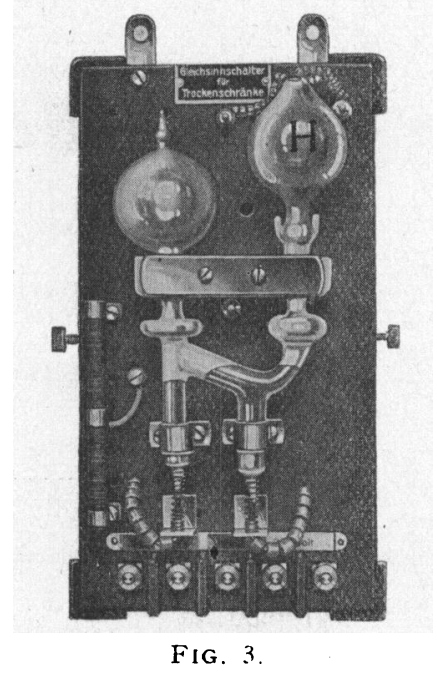

The mercurial relay of $\mathrm{W}$. C. Heraeus.

a very slight expansion under the same temperature. Further, the contact " $\mathrm{K}$ " and the two levers, $\mathrm{H} 1$ and $\mathrm{H} 2$, will be seen together with the regulation screw "R," corresponding to the thermoscale in Fig. 1. The "invar" rod " $B$ " ends with an enlargement and goes through the lever $\mathrm{H} 1$ in a groove.

The mercurial relay (Fig. 3) consists mainly of a container of quartz glass with pure mercury as the connecting medium; the main current passes through this mercurial switch to the apparatus to be heated. Further, there is mounted on the relay contacts, a resistance and a so-called heat-lamp directly connected with the switch. This heat-lamp is named " $\mathrm{H}$ " in Fig. 3.

The automatic breaking of the current takes place through a secondary current that is led through the resistance and from this to the heat-lamp "H." The switch is also filled with gas, which is warmed and drives the mercury to the left. Thus connection is obtained, and the current passes to the oven. 


\section{The Course of the Regulation}

We will suppose that all parts are mounted and the contact in place. When the regulation screw (Fig. 1) is turned completely over to the left there is no contact at " $\mathrm{K}$ " (Fig. 2) and accordingly the current is broken in the mercurial switch. Consequently no current passes to the oven. If, however, the regulating screw is turned to the right, e.g., to the line for $150^{\circ} \mathrm{C}$. the lever $\mathrm{H} 2$ (Fig. 2) is pushed against lever $\mathrm{H} 1$ and the contact is closed for the secondary current. This current passes through the relay (Fig. 3), heats the gas in the switch, the mercury is pressed over to the left and the main current is closed. As the temperature rises, the metal tube "A" in the contact thermometer (Fig. 2) will expand several times as much as the expansion of the "invar" rod "B." As this proceeds, the "invar" rod draws the lever H1 back and the connection will be broken at "K." Consequently, the secondary current is broken. The heat-lamp " $\mathrm{H}$ " in the relay will cool down. The mercury is drawn over to the right and the main current is broken. When the oven cools down $0.5^{\circ}$ to $1^{\circ} \mathrm{C}$. the contact at " $\mathrm{K}$ " (Fig. 2) is closed again. The secondary current closes the main current, and the temperature of the oven is regulated.

Regarding the thermoregulator forwarded to "Vor Frue Hospital," W. C. Heraeus recommended that the hand of the contact-thermometer scale be placed at the point for $150^{\circ} \mathrm{C}$. to obtain a temperature of $160^{\circ} \mathrm{C}$. at the mercury thermometer and not exceed this temperature. This temperature is, however, not reached till several hours after starting. To spare time, the hand is therefore first placed completely over to the right $\left(220^{\circ} \mathrm{C}\right.$.). $162^{\circ} \mathrm{C}$. at the mercury thermometer is reached after 1.5 hours (somewhat variable according to the number of instrument cases in the oven). At that time the nurse must watch the scale of the mercury thermometer at the oven closely and when $162^{\circ} \mathrm{C}$. is reached, she turns the hand down to $152^{\circ} \mathrm{C}$. on the contactthermometer scale. During the first 15 minutes the temperature rises to $165^{\circ} \mathrm{C}$. without the slightest harm to the instruments. During the following 15 minutes the temperature varies between $161^{\circ}$ and $163^{\circ} \mathrm{C}$. without any interference. The sterilization is finished, the wall contact is drawn out, the door of the oven is opened. The hand on the contact thermometer scale remains at $152^{\circ} \mathrm{C}$. until the next sterilization, when the operation again is started at $220^{\circ} \mathrm{C}$.

The automatic thermo-regulator of W. C. Heraeus is only suited for electrical heating; alternating or direct current is indifferent. But the voltage $(110$ or 220$)$ must be known. Further, one must obtain from the factory any information about the point of the scale of the contact-thermometer where the hand should be placed 
to keep the temperature between $160^{\circ}$ and $165^{\circ} \mathrm{C}$. in every individual oven.

I hope that this addition to the absolutely sure French dry air sterilization may contribute to a well-merited further use of this method, both by general surgeons and by eye operators ; especially for the latter, as the sharp point and edge are of the highest importance.

I wish to acknowledge my indebtedness to Dr. Th. Thjötta, who has undertaken all bacteriological controls mentioned in this paper and also called my attention to the automatic thermo-regulation of Heraeus, which caused me to attach this to the electrically heated dry air sterilizor of Lautenschläger.

The agent in England for W. C. Heraeus, Hanau a. Main, is : The Scientific Glass-Blowing Company, 12/14, Wright Street, Oxford Road, Manchester.

\section{THE IDENTITY OF CATARACT FORMATIONS}

BY

J. Foster, F.R.C.S.

LEEDS

THERE are still ophthalmologists in this country who prefer to use a loupe when a slit-lamp is available; possibly because they consider a loupe can reveal anything worth seeing, or possibly because of the large amount of confusing details the slit-lamp brings to light. A well used loupe may compete clinically, to a certain extent, with the slit-lamp so far as the anterior chamber is concerned, but for the lens the latter is incontestably superior. In this paper I have, therefore, made an attempt by pruning detail, and stressing practical points, to give a working classification of the types of cataract, and their appearance, as seen with the slitlamp. Nothing new is offered, and almost all the facts can be found in greater detail and different arrangement in Goulden's translation of Koby's "Slit-lamp Microscopy of the Living Eye."

Since the cause of a cataract is frequently a matter of the utmost interest from the prognostic, operative, or medico-legal aspect, the analysis of an otherwise amorphous lens opacity by the slitlamp beam is often a source of great satisfaction to the observer.

As the slit-lamp beam shines through the lens, attention must be paid, not only to the shape of the opacities, but also to the so-called "bands," and the zones of specular reflection. The former stand out as bright lines in the beam (drawn dark in Fig. 1) where areas of different refractive index, formed at different life stages, are in contact, and two Y-shaped figures are our marking 